\title{
Air-Jet Wet-Spinning of Curdlan Using Ionic Liquid
}

\author{
Shiori Suzuki,${ }^{\dagger}$ Azusa Togo, ${ }^{\dagger}$ Hongyi Gan, ${ }^{\dagger}$ Satoshi Kimura,,${ }^{\dagger} \uparrow$ and Tadahisa Iwata ${ }^{\dagger^{*}}$
}

$\dagger$ Science of Polymeric Materials, Department of Biomaterial Sciences, Graduate School of Agricultural and Life Sciences, The University of Tokyo, 1-1-1 Yayoi, Bunkyo-ku, Tokyo, 113-8657, Japan

$\$$ Department of Plant and Environmental New Resources, College of Life Sciences, Kyung Hee University, 1732 Deogyeong-daero, Giheung-gu, Yongin-shi, Gyeonggi-do, 446-701, Republic of Korea.

* Corresponding author e-mail: atiwata@g.ecc.u-tokyo.ac.jp

\section{Table of Contents}

Molecular Distributions of the Regenerated Curdlan Fibers

Fig. S1. GPC charts of curdlan before and after spinning.

Dispersion and Aggregation Behavior of Curdlan in Water and Ethanol

Fig. S2. Optical microsopic images of curdlan in water (left) and ethanol (right) at room temperature. A certain degree swelling of curdlan particles and dispersion were observed in water, whereas curdlan particles were aggregated without swelling in ethanol.

Effects of Coagulation Solvent on Mechanical Properties

Fig. S3. The tensile properties of the regenerated curdlan fibers $(D R=5)$ that were coagulated in different coagulation baths (water or ethanol). (a) Stress-strain curves and (b) elastic modulus of the fibers.

1-D X-ray Diffraction Patterns of Curdlan Powder and Regenerated Fibers

Fig. S4. 1-D X-ray diffraction patterns of the original curdlan powder and regenerated fibers that were coagulated in water at various DRs. 
Molecular Distributions of the Regenerated Curdlan Fibers.

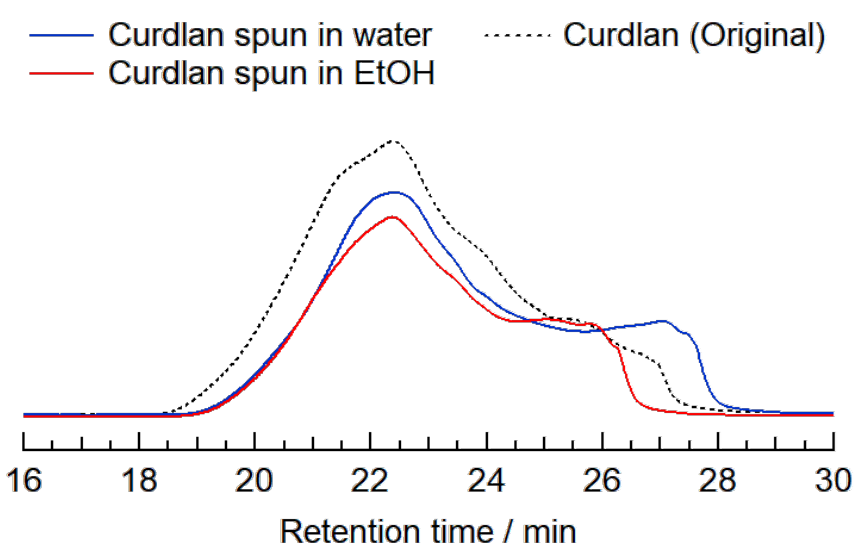

Fig. S1. GPC charts of curdlan before and after spinning.

Dispersion and Aggregation Behavior of Curdlan in Water and Ethanol.
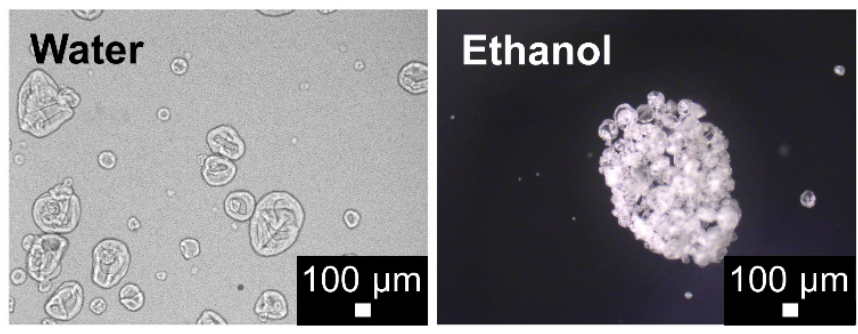

Fig. S2. Optical microsopic images of curdlan in water (left) and ethanol (right) at room temperature. A certain degree swelling of curdlan particles and dispersion were observed in water, whereas curdlan particles were aggregated without swelling in ethanol. 
Effects of Coagulation Solvent on Mechanical Properties.
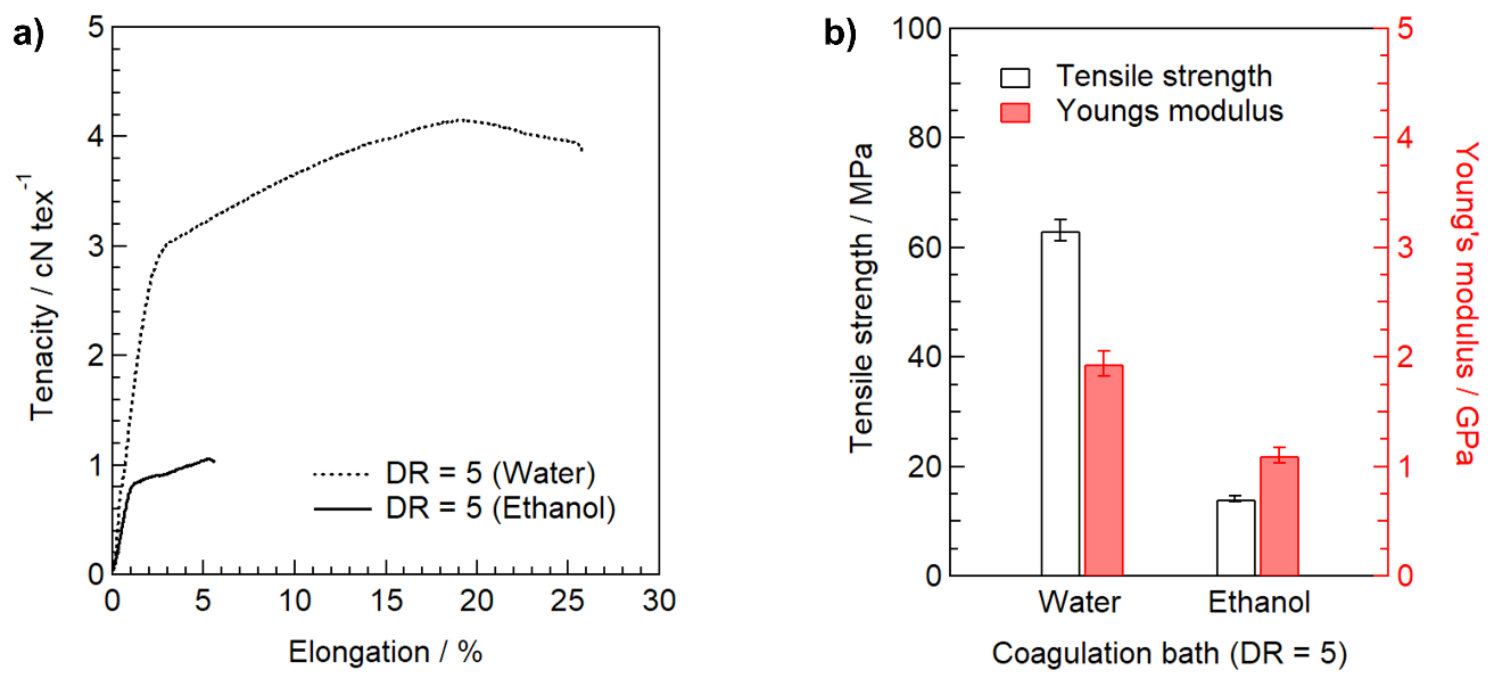

Fig. S3. The tensile properties of the regenerated curdlan fibers $(D R=5)$ that were coagulated in different coagulation baths (water or ethanol). (a) Stress-strain curves and (b) elastic modulus of the fibers.

\section{1-D X-ray Diffraction Patterns of Curdlan Powder and Regenerated Fibers.}

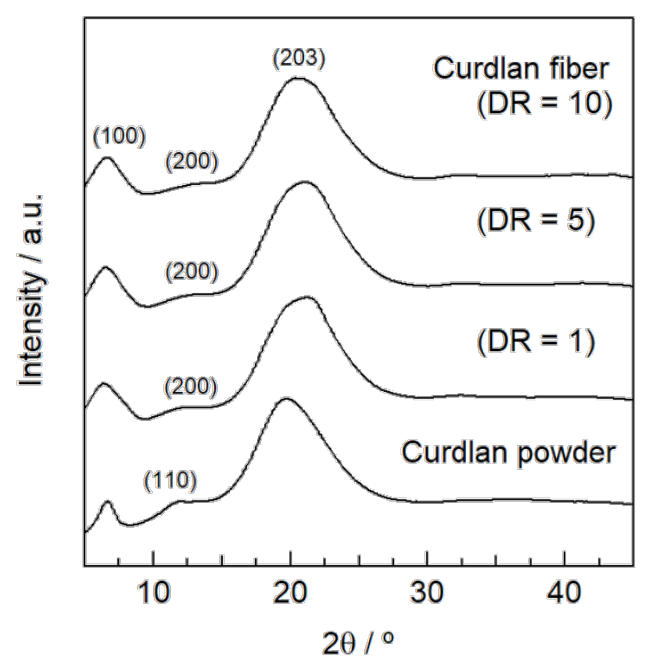

Fig. S4. 1-D X-ray diffraction patterns of the original curdlan powder and regenerated fibers that were coagulated in water at various DRs. 